\title{
A Patient-Specific Computer Model for Prediction of Clinical Outcomes in the Cerebral Circulation Using MR Flow Measurements
}

\author{
M.E. Clark ${ }^{1}$, Meide Zhao ${ }^{1}$, Francis Loth $^{1,2}$, Noam Alperin ${ }^{1,3}$, Lewis Sadler ${ }^{1}$, \\ Kern Guppy ${ }^{1}$, and Fady T. Charbel ${ }^{1}$ \\ ${ }^{1}$ Division of Neurovascular Research (CANVAS Program) \\ Department of Neurosurgery, University of Illinois at Chicago, Chicago, IL. \\ ${ }^{2}$ Department of Mechanical Engineering, University of Illinois at Chicago, Chicago, IL. \\ ${ }^{3}$ Department of Radiology, University of Illinois at Chicago, Chicago, IL. \\ m-clark4@uiuc.edu, mzhao@uic.edu, fcharbel@uic.edu, \\ Iloth@ulc.edu, alperin@uic.edu
}

\begin{abstract}
A patient-specific, computer model of the cerebral circulation to predict cerebral blood flow and pressure is presented. The model is based on a previously reported numeric model consisting of a network of distensible vessels with pulsatile flow. The enhanced model uses a sector scheme to determine the efferent resistance distribution for a specific patient. An iterative algorithm was developed to determine the patient-specific efferent resistance distribution from in vivo cerebral blood flow measurements obtained using phase contrast magnetic resonance angiography (PCMRA). In comparison with PCMRA flow measurements and clinical outcomes, the enhanced model shows its ability to predict cerebral flow well in three patients who underwent a balloon occlusion of the carotid artery. A model that accurately predicts cerebral blood flow for different treatment scenarios can provide the surgeon with an invaluable tool in the management of complex cerebral vascular disorders.
\end{abstract}

\section{Introduction}

Computer simulation of the cerebral circulation, based on fluid-dynamic relations, has been carried out for more than three decades [1-2]. It offers the convenience of predicting flows and pressures at any desired section in the vessel network. Moreover, it not only can be used to estimate the flow and pressure in both normal and disease situations, but it also can be used to predict the result of treatment procedures [3-5]. However, simulating the patient-specific cerebral circulation presents a range of challenging problems. In the fluid-dynamic area, these problems include the proper modeling of the pulsatile flow, the vessel wall distensibility, and the efferent resistance distribution. In medical imaging, there are problems associated with the automatic extraction of the patient's vasculature, accurate measurement of vessel diameters, and quantification of blood flow and pressure. The interaction of these factors makes the simulation task complex. Although much effort has been expended 
in the last three decades towards solving these problems, patient-specific fluiddynamic simulations have not yet reached the clinical utilization stage.

Cerebral circulation computer modeling can be done with various assumptions. The basic model applies the linear governing equations of steady flow and rigid vessels [1-2]. Models that incorporate the effects of pulsatile flow and distensible vessels [3-5] more accurately describe the physical behavior of blood flow in arteries. Several other cerebral circulation studies using pulsatile flow in distensible vessels have been reported [6-11]. To simulate the rupture condition of an aneurysm, Duros, et al. [5], used a 108-vessel model that not only contained the cerebral arteries but also the main systemic arteries, reasoning that they played an important role in wave propagation and overall flow distribution (Fig. 1).

In previous work [12], a computer model of cerebral blood flow was developed using angiographic determination of vessel sizes and a generic pattern for the efferent terminal resistance. This simulation was used to analyze patients with different disease states that affect the cerebral circulation. The results have correlated qualitatively with the clinical outcomes, indicating the predictive value of these simulations. In addition, the predicted blood flow patterns correlate with various test results in these patients, such as single photon emission computerized tomography (SPECT), electroencephagraphy (EEG), cerebral oxygen saturation (rSO2), and transcranial Doppler (TCD).

The generic model results, obtained using the individual patient's vessel sizes (obtained from digital subtraction angiography (DSA)) and a generic pattern of peripheral resistance, indicate satisfactory accuracy for some, but not all, vessel flows. In order to improve the simulation, patientspecific information is needed regarding the peripheral resistance distribution. To achieve this patient-specificity, the flows in certain crucial



Fig. 1 Generic 108 Yessel Model


cerebral arteries must be obtained from phase contrast magnetic resonance angiography (PCMRA). A patient-specific pattern of resistance can then be calculated. To this end, the original computer program has been modified so that all the efferents to a cerebral sector are melded into one terminal efferent vessel and a measured input flow to that sector is then used to determine its resistance. Seven such sectors have been identified in the cerebral macrovasculature. By combining the sector concept with PCMRA and DSA angiography, enhanced simulations of normal, diseased, and surgeon-modified cerebral configurations are being produced. 


\section{The Generic Model}

The behavior of the cerebral arterial network with its pulsatile flow occurring in compliant arteries is mimicked using a computer algorithm for unsteady flow in a network of elastic vessels. The primary vessels of the cerebral macrovasculature are included in the network along with a skeleton of vessels, including the heart efferent, to represent the systemic vasculature. The circle acts as an invaluable distribution center for cerebral blood but the distribution decisions are initiated in the aortic arch and subclavians.

The numeric algorithm that is used to generate the pressures and flows at all locations within the vessel network and at all times during the heart pulse period is a one-dimensional, staggered, explicit finite-difference scheme [3], based on a conservation of mass relation to calculate the pressures, and a Navier-Stokes momentum equation to calculate the flows. Not all or even most of the arteries in the cerebral circulation can be included in the model. Some judicious choice must be made as to where to terminate the model. The terminal boundary condition imposed on all efferents of the chosen network is, therefore, most crucial to the flow distributions predicted by the model and is the main thrust of this paper. A lumped parameter resistance-capacitance-resistance (RCR) combination was used in which the upstream resistance $\left(R_{1}\right)$ was set equal to the characteristic impedance of the efferent [3].

The forcing function for the model is imposed at the root of the ascending aorta and is in the form of a normal pressure-time diagram there. A set of initial conditions, required to get the numeric process started, is given by setting all nodal pressures equal to the venous pressure and all nodal flows equal to zero. Ten to fifteen pulse periods


of calculations are required to bring the pressures and flows into steady-state pulsatility. Computation time for a typical run on a $400 \mathrm{MHz}$ personal computer is approximately three minutes.

\section{Sector Model}

With the advent of PCMRA, the non-invasive measurement of cerebral artery flow has become a reality. This advance provided a means by which cerebral circulation simulations could be made more patient-specific. The crucial unknowns in such simulations are the terminal resistances of the modeled vessels. If time in the MRI machine were not a factor, the flow in each individual efferent of the model could be 
measured and a corresponding resistance could be calculated. To limit machine time and the number of flows to be determined for the patient, a sector model of the cerebral circulation was devised in which all of the terminal vessels in any sector were melded into one terminal vessel. Seven such sectors are shown in Fig.2 along with the primary and secondary flow inlets and/or outlets and with the terminal efferent for each sector. To determine this one terminal resistance, it is necessary to be able to define the flow into and/or out of the sector.



This requirement takes different forms for different sectors. For the left middle cerebral sector (LMC), there are three possible flow inlets (M1, the main middle cerebral vessel, and two small secondary collaterals or anastomoses) and one terminal resistance efferent. This single efferent takes the place of four efferents used in the original generic model (Fig.1) and has been formed by melding together these original efferents. The secondary anastomoses are not present in all cases and, when present, it is difficult to measure their flows. Therefore, the M1 flow represents the primary input to this sector and its mean flow value over a pulse period is required via PCMRA. The secondary flows are accounted for in the simulation by means of their current calculated values as described later. 
To start the computations for the sector model (Fig.3), the assumption is made that the flow (QGOAL in Fig.3) in the terminal efferent for the sector is the same as the PCMRA measured flow in the primary input flow vessel, ignoring, for this first set of calculations, the other secondary inflows or outflows. A plausible set of terminal resistances are needed to start the calculations and can be concocted from either from a steady flow model of the vessel system or from a previous similar simulation. After each (pulse) period of calculation, the program adjusts the resistance for each sector terminal efferent by multiplying its current resistance by the ratio of the current calculated terminal flow to the goal (PCMRA) flow for that terminal. This procedure would correctly adjust any one terminal flow but, since all the other terminal resistances are adjusted concurrently, errors are introduced and many adjustments are needed in subsequent periods. This process continues for up to a user-specified number of pulse periods of calculations (period_max in Fig.3). A check is made at the end of each period to see if the measured sector flow goal has been achieved by the adjustment of the resistance of the terminal efferent. If all seven sector flow goals are achieved within the period limit, the program proceeds to the next step. If the goals are not met after the maximum number of periods, the program starts over with the current efferent resistances and the original initial conditions of zero flow and venous pressure at all nodes in all vessels.

The next step in the procedure is the adjustment of the flow goals (QGOALs in Fig.3) so as to account for the secondary inflows and/or outflows. Since all vessels in the model are involved in the calculations discussed above, the secondary flows have been continually updated in this process. In this set of calculations, the flow in the terminal efferent is set equal to the primary PCMRA flow adjusted for the flows in the secondary inlets and/or outlets. For the left middle cerebral sector, this amounts to adjusting the M1 flow for the inflow or outflow contributions of the two secondary anastomoses. For the basilar sector, the contributions of the posterior communicating arteries and the two secondary anastomoses must be considered with proper signs. For the left external carotid sector, the ophthalmic flow must be combined with the left external carotid PCMRA flow in subsequent calculations. These adjustments are made only at the beginning of a period_max run, which can be cut short if all sector flow goals are met. When a user-specified number of adjustments have been made, the program ends.

At this stage of the simulation, a patient-specific set of resistances has been generated from patient DSA angiograms and measured PCMRA sector flows. This resistance pattern can now be assumed to be invariant in additional simulations of this patient. Modifications in system configuration can be made, say, by inserting a bypass vessel between the external and internal carotid arteries or by vessel occlusion in the treatment of an aneurysm. The neurosurgeon can now play "what if?" games with the computer program to plan the best strategies for his/her treatment of the patient.

\section{Cerebral Blood Flow Quantification}

Flow quantification with PCMRA provides a powerful tool to non-invasively analyze blood flow [15]. It has been shown to do well in assessing both in vitro and in vivo flow velocities and volumetric flow rates (VFR) [16]. Accuracy of blood flow measurements is influenced by many MR parameters : among these are the partial volume [17] and the curved flow effect [18]. 
A three-dimensional (3D) vessel localization algorithm has been developed by Zhao, et al. [18] for the determination of slice selections and slice orientation to improve the accuracy of flow measurement. The 3D localizer was developed based on a 1.5 Tesla MRI General Electric imager. A 3D time of flight MRA covering the circle of Willis is performed and the images are extracted and transferred to a SGI workstation. The 3D surface rendering of the vasculature is then reconstructed from these images using a marching-cube algorithm [18]. The coordinates obtained from the $3 \mathrm{D}$ localization based on the $3 \mathrm{D}$ surface rendering specify the position of an oblique cine PCMRA scan. A cine PCMRA is then performed based on those coordinates. The following parameters were used: TR, $33 \mathrm{~ms}$; TE, minimum; flip angle, 30; number of excitations, 2; field of view, $16 \mathrm{~cm}$; image matrix, 256 x 128; number of phases, 32; slice thickness and velocity encoding (VENC) are $5 \mathrm{~mm}$ and $100 \mathrm{~cm} / \mathrm{sec}$ for carotids, VA, and BA, and $4 \mathrm{~mm}$ and $70 \mathrm{~cm} / \mathrm{sec}$ for MCA and ACA. The vessel area is semi-automatically determined though a newly-developed interface on a SGI workstation [18]. The velocity and volumetric flow rate (VFR) can then be calculated over the time course of the pulse period.

\section{Presentation of Results - Case Studies}

To exhibit the efficacy and abilities of the sector model of the cerebral circulation, three cases relating to the balloon occlusion test (BOT) used in the treatment of nonoperable cerebral aneurysms will be presented and discussed. When an aneurysm is to be treated by occluding the vessel to which it is attached, a temporary occlusion of the vessel is made with a balloon to test the clinical outcome of the patient to permanent occlusion. Since the BOT is not without risk, the patient would benefit if a BOT computer simulation could be substituted. The three cases presented for discussion are intended to show how well the model predicts the BOT outcome.

Case 1. A 51-year-old white male presented with a two-month's history of progressively worsening left pulsatile tinnitus. Angiogram and PCMRA studies showed that both the left and right posterior communicating arteries and the A1 segment of the left anterior cerebral artery were small. The left internal carotid artery contained a large aneurysm and a stenosis of 60 percent. These data were used as Baseline in the computer simulation. The patient subsequently passed a BOT. A permanent occlusion of the LICA was made with balloons trapping the diseased distal cervical segment of the LICA.

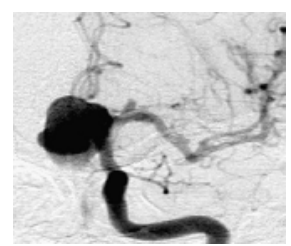

(a)

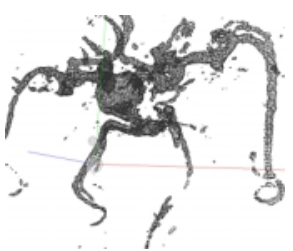

(b)

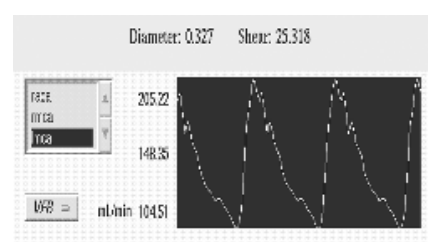

(c)

Fig. 4 Angiogram (a), 3D Localizer Image (b), and Flow Waveform (c) for Case 2. 


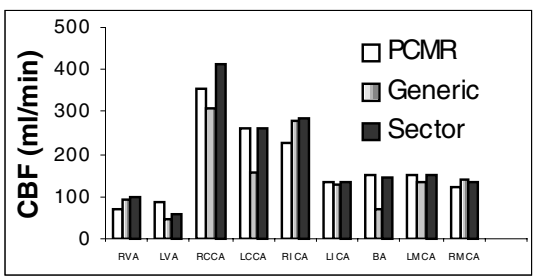

(a)



(c)

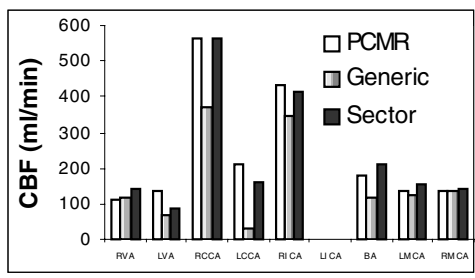

(b)

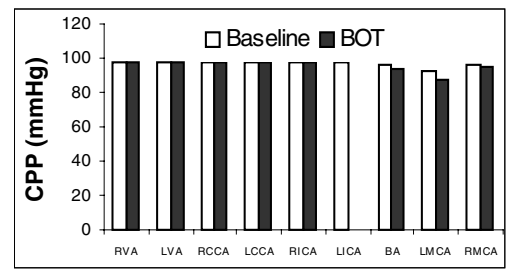

(d)

Fig.5 Results for Case 1: (a) Comparison of CBF (Cerebral Blood Flow) at Baseline and (b) post-BOT between PCMRA and simulations from Generic and Sector Models; Comparison of CPP (Cerebral Perfusion Pressure) between Generic (c) and Sector (d) simulations at Baseline and BOT

Case 2. A 56-year-old black female presented with a subarachnoid hemorrhage. Angiogram and PCMRA studies showed multiple aneurysms including a giant aneurysm at the left superior hypophyseal artery as well as a 40 percent stenosis in the left internal carotid artery. The patient failed the BOT under hypotensive challenge, becoming hemiparetic and aphasic during the test. SPECT also showed a massive difference between the two hemispheres with a significant decreased relative blood flow in the left hemisphere involving the frontoparietal, occipital and temporal lobes. A PCMRA CBF study was performed before the BOT. These data were used in the Baseline simulation.

Case 3. A 71-year-old white female presented with persistent headache. Angiogram and PCMRA studies showed a large right carotid-ophthalmic aneurysm as well as occlusions of both left and right posterior communicating arteries, occlusion of the anterior communicating artery, and a 40 percent stenosis in the left anterior cerebral artery. These data were considered as the Baseline in the computer simulation. A PCMRA CBF study was performed before the BOT. During the BOT in the right internal carotid artery, an immediate failure ensued when left side hemiparesis developed.

A typical angiogram, 3D localizer image, and flow waveform are presented in Fig. 4. The PCMRA and simulation results are shown in the form of bar graphs in Fig. 5 for Case 1 and in Fig.6 for Cases 2 and 3. These results will be discussed in detail in the next section. 


\section{Discussion and Conclusions}

The purpose of this paper is to demonstrate the efficacy of a novel simulation technique, the sector model, in the patient-specific prediction of clinical outcomes accompanying surgical intervention. The specific intervention considered here is the treatment of inoperable aneurysms by permanent occlusion of a carotid artery. The patient's tolerance of such an occlusion is determined by temporarily occluding the artery, the balloon occlusion test (BOT). Since this test is not without risk, it would be beneficial for the patient to substitute a valid computer simulation for it.

The PCMRA flow measurements along with the sector and generic model results are displayed in Fig. 5 and 6. For case 1, the Baseline results in Fig. 5a show excellent agreement between the PCMRA flow and the sector model results for most vessels. This result was expected since the PCMRA flow measurements were used in the RCR adjustments to satisfy the iterative process of Fig. 3. Once the RCR pattern had been determined for this patient, it was used independently to predict the post-BOT results shown in Fig.5b. Here again, the agreement between the PCMRA and the sector model results is excellent (with the possible exception of the LVA). The generic model compared well with the PCMRA measurements for some vessels, but not as consistently as the sector model. For cerebral perfusion pressure (CPP) results in Fig. $5 \mathrm{c}$ and $5 \mathrm{~d}$, both sector and generic models give comparable predictions for Baseline and BOT with no dramatic pressure drops during the test. Since the patient passed the test, these results are also compatible with the clinical outcome.

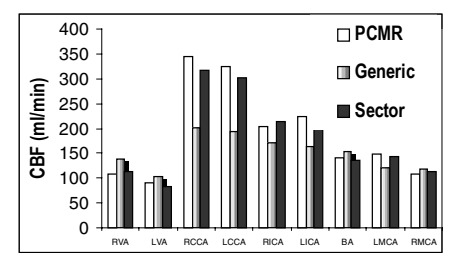

(a) Case 2

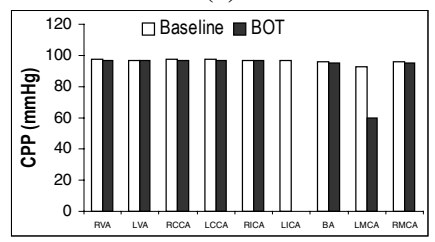

(c) Case 2

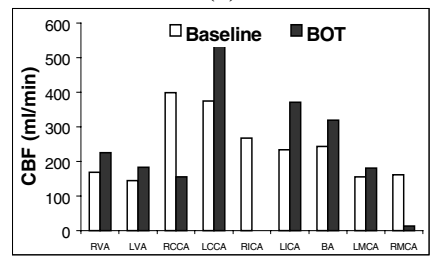

(e) Case 3

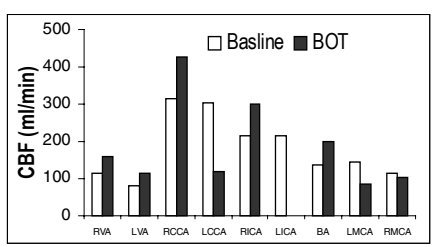

(b) Case 2

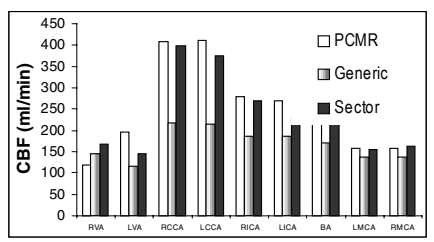

(d) Case 3

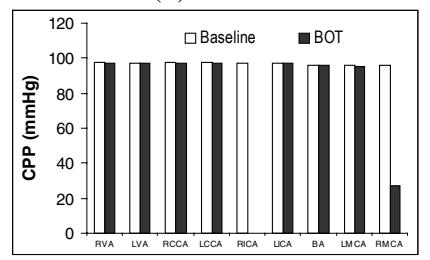

(f) Case 3

Fig. 6. Results for Case 2 (a)-(c) and Case 3 (d)-(f): Comparison of CBF at Baseline in Case 2 (a) and Case 3 (d) between PCMRA and simulations from Generic and Sector Models; Comparison of CBF in Case 2 (b) and Case 3 (e) and CPP in Case 2 (d) and Case 3 (f) between simulations at Baseline and BOT 
Similar results were obtained for Cases 2 and 3 for Baseline CBF as shown in Fig. 6a and 6d. Since both patients failed the BOT test, there are no post-BOT PCMRA data to compare with the simulation results. Therefore, only calculated Baseline and BOT CBF values are shown in Fig.6b and 6e for Cases 2 and 3, respectively. These cases have drastically different flow patterns in the carotid vessels (RCCA, LCCA, RICA, LICA) because of the balloon closure of the LICA and RICA in Cases 2 and 3, respectively. For the BOT simulation, the reduction in flow values for the LMCA in Case 2 and the RMCA for Case 3 in comparison with the Baseline values is compatible with the clinical outcome of BOT failure. A similar result for CPP values for these arteries is shown in Fig. 6c and 6f. The reduction in flow and pressure for Case 2 was not as pronounced as that for Case 3 because Case 2 failed the BOT only under hypotensive challenge. In Case 3, the reduction in flow is nearly 100 percent and the pressure is reduced to venous pressure levels in the RMCA.

For Case 3, when the seven-sector model was used to simulate the BOT, an anomaly was discovered in the AC sector. All possible inflow paths to the RMC and AC sectors were seemingly blocked by the occlusions indicated in Section 5. However, the sector model found the one unblocked path. That path started by following the LAC up to the junction of the terminal efferent. Since only part of the available LAC flow was needed to satisfy that efferent, the rest passed down the RAC and went on to satisfy the RMC sector. To correct this implausible solution and to prevent the anomaly, an eight-sector model was used with two separate AC sectors. However, the resulting RAC and RMC sectors had no connection to the forcing function in the ascending aorta except for minor contributions from the small ophthalmic and two secondary anastomoses. The results of the new simulation showed essentially zero flow and venous pressure levels in these sectors. The immediate left side hemiparesis in this patient is adequately explained by these simulation results.

In conclusion, patient-specificity has been achieved in the simulation and analysis of the three BOT patients. These results demonstrate a significant advance in computer modeling of the cerebral circulation through the introduction of the sector model. While the present study only examined three cases, the results clearly demonstrate the working hypothesis of this sector model. Further work using the model in a variety of surgical interventions and with a large population of patients is necessary before it is ready for clinical use. Current work is underway to obtain these validation data. Many improvements are envisioned for future models: the forcing function can be made more patient-specific; the systemic portions of the model can be made into sectors and governed by PCMRA measurements; and the autoregulatory aspects of the cerebral arterioles can be added to the simulation. In addition, the model can be generalized to other regions of the cardiovascular system like the coronary circulation or the circulation in the extremities. A model that can accurately predict cerebral blood flow will provide an invaluable tool for the surgeon in managing complex cerebral problems. 


\section{References}

1. Himwich, W.A, Knapp, F.M., Wenglarz, R.A., Martin, J.D., and Clark, M.E.: The circle of Willis as simulated by an engineering model, Arch. of Neurology, (1965) 13: 164-172

2. Clark, M.E., Himwich, W.A., and Martin, J.D.: Simulation studies of factors influencing the cerebral circulation, Acta Neurol. Scandinav., (1967) 43: 189-204

3. Kufahl, R.H., and Clark, M.E.: A circle of Willis simulation using distensible vessels and pulsatile flow, J. of Biomechanical Engineering, (1985) 107: 112-122

4. Clark, M.E., Kufahl, R.H., and Zimmerman, F.J.: Natural and surgically-imposed anastomoses of the circle of Willis, Neurological Research, (1989) 11: 217-230

5. Duros, J., Clark, M.E., Kufahl, R.H., and Nadvornik, P.: On the rupture of an aneurysm, Neurological Research, (1991) 13: 217-223

6. Ling, S.C., and Atabek, H.B.: A nonlinear analysis of pulsatile flow in arteries, J. of Fluid Mechanics, (1972) 55: 493-511

7. Cooper, L.: Pulsating flow in a network with application to the circle of Willis, (1970) Ph.D. thesis, Washington University, St.Louis, MO

8. Chao, J.C., and Hwang, N.H.: Function dynamics of the circle of Willis, J. of Life Science, (1972) 2: 3-81

9. Himwich, W.A., and Clark, M.E.: Simulation of flow and pressure distributions in the circle of Willis. In: J. Cervos-Navarro, eds., Pathology of Cerebral Microcirculation, NY, Walter de Gruyter, (1974) 140-152

10. Clark, M.E., and Kufahl, R.H.: Simulation of cerebral macrocirculation. In: Proc of $1^{\text {st }}$ Int. Conf. Cardiovascular System Dynamics, MIT Press, Boston (1978) 380-390

11. Hillen, B., Gaasbeek, T., and Hoogstraten, H.W.: A mathematical model of the flow in the posterior communicating arteries, J. of Biomechanics, (1982) 15: 441-448

12. Charbel, F.T., Misra, M., Clark, M.E., and Ausman, J.I: Computer simulation of cerebral blood flow in Moyamoya and the results of surgical therapies, Clinical Neurology and Neurosurgery, (1997), Suppl.2.

13. Charbel, F.T., Clark, M.E., Misra, M., Hannigan, K., Hoffman, W.E., and Ausman, J.I.: The application of a computerized model of the cerebral circulation in skull base surgery. $2^{\text {nd }}$ Intern. Skull Base Congress, San Diego, (1996) 210

14. Raines, J.K., Jaffrin, M.Y., and Shapiro, A.H.: A computer simulation of the human artery system, Proc. of the 1971 Summer Computer Conference, (1971), 2, 171-178

15. Moran, P.R.: A flow velocity zeugmatographic interlace for NMR imaging in humans, Magnetic Resonance Imaging, (1982) 1, 197-203

16. Hofman, M.B.M., Visser, F.C., Vanrossum, A.C., Vink, G.Q.M., Sprenger, M., and Westerhof, N.: In Vivo validation of magnetic resonance blood volume flow measurements with limited spatial resolution in small vessels, Magnetic Resonance in Medicine, (1995), 33, 778-784

17. Tang, C., Blatter, D., and Parker, D.: Accuracy of phase-contrast flow measurements in the presence of partial-volume effects, JMRI, (1993) 3, 377-385

18. Frank, L., and Buxton, R.: Distortions from Curved Flow in magnetic Resonance Imaing, JMRI, (1992) 2, 82-93

19. Zhao, M., Charbel, F.T., Loth, F., and Alperin, N.: Improved Phase Contrast Quantification by 3D Vessel localization, (1998) Xth Annual International Workshop on Magnetic Resonance Angiography, Park City, Utah; Submitted to JMRI. 